https://doi.org/10.15407/ujpe65.10.881

V.A. STEPHANOVICH, ${ }^{1}$ YU.G. SEMENOV ${ }^{2}$

${ }^{1}$ Opole University, Institute of Physics

(Oleska 48, 55-052, Opole, Poland)

${ }^{2}$ V. Lashkaryov Institute of Semiconductor Physics, Nat. Acad. of Sci. of Ukraine

(41, Nauky Prosp., Kyiv 03680, Ukraine)

\title{
THE MAGNETIC DOMAIN \\ STRUCTURE PROPERTIES IN DILUTED MAGNETIC SEMICONDUCTORS
}

\begin{abstract}
We present a comprehensive analysis of the domain structure formation in the ferromagnetic phase of diluted magnetic semiconductors (DMS) of the p-type. Our analysis is carried out on the base of the effective magnetic free energy of DMS calculated by us earlier. This free energy, substituting DMS (a disordered magnet) by an effective ordered substance, permits us to apply the standard phenomenological approach to the domain structure calculation. Using the coupled system of Maxwell equations with those obtained by the minimization of the free energy functional, we show the existence of the critical ratio $\nu_{\mathrm{cr}}$ of concentration of charge carriers and magnetic ions such that the sample critical thickness $L_{\mathrm{cr}}$ (such that the sample is monodomain at $L<L_{\mathrm{cr}}$ ) diverges as $\nu \rightarrow \nu_{\mathrm{cr}}$. At $\nu>\nu_{\mathrm{cr}}$, the sample is monodomain. This feature makes DMS different from conventional ordered magnets, as it gives a possibility to control the sample critical thickness and the emerging domain structure period by a variation of $\nu$. As the concentration of magnetic impurities grows, $\nu_{\mathrm{cr}} \rightarrow \infty$, restoring a conventional behavior of ordered magnets. Above facts have been revealed by the examination of the temperature of the transition to an inhomogeneous magnetic state (stripe domain structure) in the slab of a p-type DMS with finite thickness L. Our theory can be easily generalized for an arbitrary disordered magnet.
\end{abstract}

Ke ywords: magnetic domain structure, diluted magnetic semiconductor.

\section{Introduction}

In 1976, the giant enhancement of the magnetic field effect on exciton spectra in a diluted magnetic semiconductors (DMS) had been discovered at the Ukrainian Institute of Physics in the research team headed by S.M. Ryabchenko. The effect was explained by the collective influence of spin-polarized magnetic ions on the electronic states via the carrierion exchange interaction [1]. Very soon, the promotion of this unusual effect became a main stream in multiple physical labs in Europe and USA. It was not surprising that such active study of DMS evoked the idea to explore the inverse effect of band carriers on the spin polarization of magnetic ions that can ultimately mediate the ferromagnetic phase transition. The theory of this effect had been put forward by Pashitskii and Ryabchenko [2]. However, Pashitskii and Ryabchenko's initial approach to this prob-

(C) V.A. STEPHANOVICH, YU.G. SEMENOV, 2020

ISSN 2071-0194. Ukr. J. Phys. 2020. Vol. 65, No. 10 lem exploited the relatively weak effect of conduction band electrons that was too faint to obtain the ferromagnetic state of DMS at reasonably high temperatures [2]. That is why the potentially seminal paper remained unheeded up to the Ohno et al. effort to enhance the ferromagnetic correlations in $p$-type DMS (see [3] and references therein). Herewith, the latter works focused on developing the homogeneous magnetic states. On the other hand, engineered magnetic domains with designed shapes have proven to be useful in many applications and in spintronics, in particular $[3,4]$. Since DMSs are the disordered magnets, they demonstrate a rich variety of magnetic properties that have a great potential to manipulate the domain structure in the magnetically ordered phase to obtain the magnetic properties useful for applications [4-8].

The structure of domains and domain walls in conventional ordered magnets has been well studied both experimentally and theoretically several decades ago 
(see, e.g., $[9,10]$ and references therein). The first quantitative theory of domain structure in ordered ferromagnets had been suggested by Landau and Lifshits in their classical paper [11] (see also [9]). The second step had been done by Shirobokov [12], who found the periodic distribution of magnetic moments in a magnetically ordered crystal, neglecting, however, the inhomogeneous distribution of a demagnetization field deep inside a sample. It was shown further (see $[10,13]$ and references therein) that such approach is valid only at sufficiently low temperatures ( $T \ll T_{c}$, where $T_{c}$ is the phase transition temperature), where the demagnetizing field is localized near the crystal surface, and the domain walls are sufficiently thin. In that case, the domains are large, and the magnetization inside them equals to that of a bulk "domainless" sample. As the temperature grows approaching $T_{c}$, the demagnetizing field penetrates deeply inside a crystal. In other words, the magnetization inside domains begins to depend on that field and vice versa - the demagnetization filed depends on the above magnetization so that the problem becomes self-consistent. It was shown in $[10,13]$ that the properties of the domain structure of any magnetically ordered substance are completely determined by the joint solution of Maxwell equations for a demagnetization field and those obtained by the minimization of the corresponding Landau free energy functional of a magnet. The shape of a sample is determined by corresponding boundary conditions. This approach has already become standard for the calculation of physical properties of the domain structure of ordered magnets.

When a magnet is disordered, which is the case for diluted magnetic semiconductors (DMSs), the application of the above formalism is impossible, as there is no corresponding free energy functional. In our opinion, this is the cause for why in the papers, where the ferromagnetism in DMS has been reported and investigated, the important question about their domain structure has not been addressed (see $[5,14]$ ). The characteristics of a domain structure in DMS films of the III-V type have been investigated in $[7,8]$. For instance, in Ref. [8], the conventional stripe-shaped domain structure has been observed in $\mathrm{Ga}_{0.957} \mathrm{Mn}_{0.043} \mathrm{As}$. As we have mentioned above, the main problem in theoretical descriptions of the DMS domain structure in the mentioned papers was the lack of a suitable "continuous" free energy func- tional. Such free energy functional had been derived by us previously [15], where our starting point was either the Ising or Heisenberg model of DMS. To derive the free energy functional, we use the procedure of self-consistent effective averaging over the DMS magnetic impurity ensemble. As a result, we obtain the effective free energy functional, where the coefficients before the magnetization powers contain not only the temperature, but the concentration of magnetic ions as well. In other words, our free energy function gives a "mapping" of the initial disordered substance onto some effective ordered magnet, where the effects of dilution (disorder) are considered as a variable concentration of magnetic ions.

In the language of a phenomenological theory of magnetism, this functional corresponds to the so-called homogeneous exchange part of the total phenomenological free energy of DMS. To describe the domain structure properties, these contributions should be completed by the inhomogeneous exchange and magnetic anisotropy energies. This can be done in a standard phenomenological way. Namely, it was demonstrated experimentally (see [14] and references therein) that cmagnetic anisotropy exists in DMSs of the $(\mathrm{Ga}, \mathrm{Mn})$ As type. At the same time, it was demonstrated in Ref. [14] that unstrained samples (which can be well described by the Heisenberg model) have easy plane magnetic anisotropy, while uniaxially strained samples (Ising model) have an anisotropy of the easy axis type.

It is well known (see $[9,17])$ that, at low temperatures, the domain pattern formation is primarily due to the rotation of the magnetization vector with constant modulus, being the saturation magnetization $M_{0}$. On the contrary, for the temperatures close to $T_{c}$, this structure is formed by a variation of the modulus of $\mathbf{M}$, rather than its rotation. This means that the homogeneous exchange part of the magnetic energy of DMS will only contribute to its domain structure near $T_{c}$. At low temperatures, the influence of a disorder on the domain structure of DMS is small, so that it will resemble very much the domain structure of conventional magnetically ordered substances.

Having the complete free energy functional of DMS, we can apply the above standard approach to calculate the properties of the domain structure of DMS in the entire temperature range, where the ferromagnetism exists. However, far from the phase transition temperature, the solution of the correspondent non- 
linear partial differential equations can be done only numerically. That is why in the present paper, we suggest a theory of inhomogeneous magnetic state (stripe domain structure) in the DMS slab in a vicinity of the ferromagnetic phase transition temperature. We analyze a sample with finite thickness $L$. We show that the impurity character of ferromagnetism in DMS results in a substantial narrowing of the region of temperatures and sample thicknesses, where a domain structure exists. For example, in the disordered magnetic substances under consideration, the domain structure appears at some threshold value $L_{\mathrm{tr}}$, depending on the ratio $\nu$ of the concentrations of charge carriers and magnetic ions $\left(n_{c}\right.$ and $n_{i}$, respectively, so that $\left.\nu=n_{c} / n_{i}\right)$. This can be approximated by a simple analytic dependence $L_{\mathrm{tr}} \propto\left|\nu-\nu_{\mathrm{cr}}\right|^{-b}$, where $\nu_{\mathrm{cr}}$ and $b$ depend on the DMS model (Heisenberg or Ising one). This is shown in Fig. 1, where the region of parameters ensuring the existence of the domain structure in a disordered substance (DMS) is much smaller than that for an ordered magnet. The area of that region is determined by the value of $\nu-$ an additional parameter which is absent in ordered magnets. This effect makes the domain structure of DMSs qualitatively different from that of conventional ordered magnets. The developed formalism can be easily extended to a wide temperature range and to thin DMS films. It can also be applied to the virtually any kind of magnetically disordered materials.

\section{Formalism}

Consider a slab-shaped sample of DMS with thickness $L$ (Fig. 1, lower panel). Let the $z$ axis is the magnetic anisotropy one (and the $x y$ plane is the anisotropy one for the Heisenberg model). The phenomenological free energy of DMS near $T_{c}$ can be written in the form (see, e.g., [10])

$F=\int\left\{\frac{1}{2} \alpha(\nabla \mathbf{M})^{2}+f_{\mathrm{AN}}^{\mathrm{P}}+f^{\mathrm{P}}(M)-\frac{1}{2} \mathbf{M H}_{\mathrm{D}}\right\} d^{3} x$

where $\mathbf{M}$ is the magnetization vector, $\alpha$ is the inhomogeneous exchange constant, $\mathbf{H}_{\mathrm{D}}$ is a demagnetization field, $f_{\mathrm{AN}}^{\mathrm{P}}$ and $f^{\mathrm{P}}(M)$ are, respectively, the (model-dependent with $\mathrm{P}=\mathrm{H}$, I, where $\mathrm{H}$ and I stand for the Heisenberg and Ising models, respectively) anisotropy and homogeneous exchange energies. For the Heisenberg model (easy plane aniso-

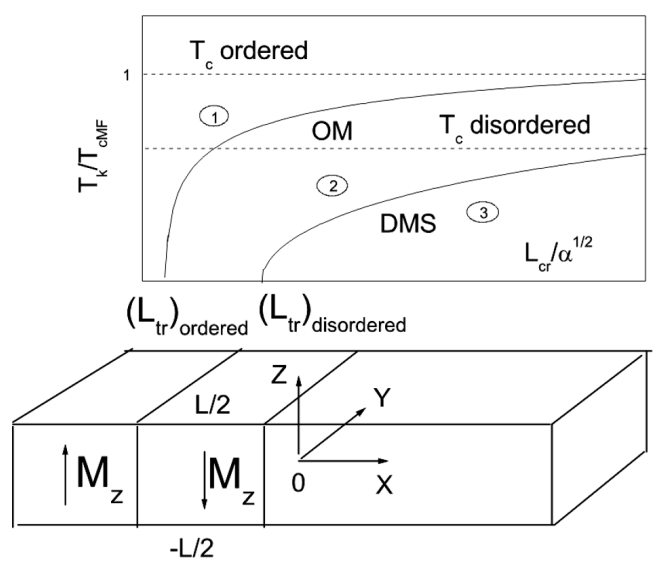

Fig. 1. Upper panel - schematic temperature-thickness phase diagram of the DMS. $T_{k}$ is the temperature of a phase transition into the domain state. Region 1 corresponds to the paramagnetic phase for both ordered (OM) and disordered (DMS) magnets, region 2 - paramagnetic phase for DMS and the domain state for OM, region 3 - domain state for both cases. Horizontal asymptotes correspond to $\tau_{c}(\nu)=T_{c} / T_{\mathrm{cMF}}, T_{c}$ is the temperature of a phase transition into the ferromagnetic homogeneous (i.e. domainless) state. Lower panel shows the geometry of the sample

tropy), we have

$f_{\mathrm{AN}}^{\mathrm{H}}=\frac{1}{2} \beta M_{x}^{2}, \beta>0$

$f^{\mathrm{H}}(m)=\frac{1}{2} m^{2}\left(1-2 A_{1}^{\mathrm{H}}\right)+\frac{1}{20} m^{4} A_{3}^{\mathrm{H}}+\ldots$,

$A_{n}^{\mathrm{H}}=\int_{0}^{\infty} \mathcal{B}_{1 / 2}^{\mathrm{H}}(\pi t) e^{-\mathcal{F}_{0}(t / 2 T)} \mathcal{F}_{1}^{n}\left(\frac{t}{2 T}\right) d t$

where $m=|\mathbf{m}|, \mathbf{m}=\overline{\langle\mathbf{S}\rangle} / S, S$ is a magnetic ion spin. Bar means the averaging over a spatial disorder in the magnetic ion subsystem of DMS, while angular brackets mean the thermal averaging, see Ref. [16] for details. The relation between $m$ and $M$ in this case is usual: $m=M / M_{0}$, where $M_{0}$ is saturation magnetization.

For the Ising model, we have the easy axis anisotropy so that

$f_{\mathrm{AN}}^{\mathrm{I}}=\frac{1}{2} \beta\left(M_{x}^{2}+M_{y}^{2}\right), \beta>0$,

$f^{\mathrm{I}}(m)=\frac{1}{2} m^{2}\left(1-A_{1}^{\mathrm{I}}\right)+\frac{1}{24} m^{4} A_{3}^{\mathrm{I}}+\ldots$,

$A_{n}^{\mathrm{I}}=\int_{0}^{\infty} \mathcal{B}_{1 / 2}^{\mathrm{I}}(\pi t) e^{-\mathcal{F}_{0}(t / 2 T)} \mathcal{F}_{1}^{n}\left(\frac{t}{2 T}\right) d t$. 
Here
$\mathcal{B}_{1 / 2}^{\mathrm{H}}(x)=\frac{1+x \operatorname{coth} x}{3 \sinh x}, \quad \mathcal{B}_{1 / 2}^{\mathrm{I}}(x)=\frac{1}{\sinh x}$,

$\mathcal{F}_{0}(x)+i \mathcal{F}_{1}(x)=\int_{V} n(\mathbf{r})\left[1-e^{-i J(\mathbf{r}) x}\right] d^{3} r$,

$\mathcal{F}_{0,1}$ are the real and imaginary parts of the Fourier transform of the distribution function of a random magnetic field acting among the magnetic impurities in DMS, $J(\mathbf{r})$ is the $\mathbf{r}$-dependent exchange integral in the microscopic versions of the Ising or Heisenberg spin Hamiltonian, see $[15,16]$ for details. In addition, $n(\mathbf{r})$ is a spatially nonuniform concentration of magnetic ions. Index $1 / 2$ in the functions (8) means that the free energies (3) and (6) have been derived for spin $1 / 2$ (see [15]). However, our analysis shows that while these results give the qualitative answer for any spin, they can be generalized to the latter case.

Note that, in our random field method (see Refs. $[15,16])$ mean field asymptotics formally corresponds to the limit $n_{c} / n_{i} \rightarrow 0$ corresponding to the case of ordered substance, where all lattice sites are occupied by magnetic ions. In this approximation, the expression for the phase transition temperature into a spatially homogeneous domainless state reads [14]

$T_{\mathrm{cMF}}=\frac{1}{4} \int n(\mathbf{r}) J(\mathbf{r}) d^{3} r$.

The equilibrium distribution of the magnetization in DMS can be obtained from the equation of state

$\frac{\delta F}{\delta \mathbf{m}}=0$

augmented by the Maxwell equations

$\operatorname{rot} \mathbf{h}=0, \quad \operatorname{div}(\mathbf{h}+4 \pi \mathbf{m})=0$

along with boundary conditions for the slab geometry

$$
\begin{aligned}
& \left.h_{x}\right|_{z= \pm \frac{L}{2}}=\left.h_{x}^{(e)}\right|_{z= \pm \frac{L}{2}},\left.\quad \frac{\partial \mathbf{m}}{\partial z}\right|_{z= \pm \frac{L}{2}}=0 \\
& {\left[h_{z}+4 \pi m_{z}\right]_{z= \pm \frac{L}{2}}=\left.h_{z}^{(e)}\right|_{z= \pm \frac{L}{2}}}
\end{aligned}
$$

where $\mathbf{h}=\mathbf{H}_{\mathrm{D}} / M_{0}, \mathbf{h}^{(e)}$ is a demagnetizing field in vacuum. It was shown in Ref. [10] that, for sufficiently thick slabs and $\beta<4 \pi$, the following equation for the distribution of the magnetization in DMS can be derived from the equation of state (11) with regard for

$\mu_{\perp} \frac{\partial^{2}}{\partial x^{2}}\left(\alpha \frac{\partial^{2} m_{z}}{\partial x^{2}}-b_{\mathrm{P}} m_{z}-c_{\mathrm{P}} m_{z}^{3}\right)-4 \pi \frac{\partial^{2} m_{z}}{\partial z^{2}}=0$, where $\mu_{\perp}=1+4 \pi / \beta, b_{\mathrm{H}}=1-2 A_{1}^{\mathrm{H}}, c_{\mathrm{H}}=A_{3}^{\mathrm{H}} / 5, b_{\mathrm{I}}=$ $=1-A_{1}^{\mathrm{I}}, c_{\mathrm{I}}=A_{3}^{\mathrm{I}} / 6$. It can be shown that, at $T<T_{c}$ (ferromagnetic phase), the functions $b_{\mathrm{I}, \mathrm{H}}(T)<0$. Note that the different forms of anisotropy energies for the Heisenberg (Eq. (2)) and Ising (Eq. (5)) models under the above suppositions do not influence the form of Eq. (14).

It had been shown in $[10,13]$ that the transition from the paramagnetic phase to the ferromagnetic one with a domain structure (domain state) occurs as a phase transition of the second kind. This means that the magnetization (order parameter) is infinitesimal at the phase transition point and its close vicinity. Therefore, to determine the transition temperature $T_{k}$ to the domain state, it is sufficient to consider the linearized version of Eq. (14). We look for its solution in the form

$m_{z}=A \cos q z \cos k x$.

Here, $\cos q z$ determines the spatial inhomogeneity along the $z$ direction, while $\cos k x$ defines a "linear" domain structure (in the $x$ direction, so that the domain walls lie in the $y z$ plane) with the period $d=2 \pi / k$.

The substitution of solution (15) into the linearized version of Eq. (14) gives the equation relating $q$ and $k$ :

$q^{2}=\frac{\mu_{\perp}}{4 \pi} k^{2}\left(b_{\mathrm{P}}+\alpha k^{2}\right)$.

To obtain the dependence of $T_{k}$ on the sample thickness, we need one more equation relating $b$ (and $A$ and $T_{k}$, see Eqs. (4), (7)), $k$, and $q$. Such equation can be obtained, by substituting Eq. (15) into the boundary conditions (13) with regard for the vacuum solutions $h_{z}^{(e)}=C \exp (-k|z|) \cos k x, h_{x}^{(e)}=$ $=C \exp (-k|z|) \sin k x$. It reads

$\tan \frac{q L}{2}=\frac{\mu_{\perp} k}{q}$.

Equations (16) and (17) constitute a closed set of equations for the instability temperature $T_{k}$ and equilibrium domain structure period (width of domain stripes) as a function of the sample thickness $L$ and the concentration ratio $\nu$. Equations (16) and (17) can be reduced to a single equation describing the dependence of $b_{P}$ on $y=k \sqrt{\alpha}$ :

$y \sqrt{\frac{\left(-\mu_{\perp}\right)}{4 \pi}\left(b_{\mathrm{P}}+y^{2}\right)}=\pi n+\frac{2}{\eta} \arctan \sqrt{\frac{\left(-4 \pi \mu_{\perp}\right)}{b_{\mathrm{P}}+y^{2}}}$,

ISSN 2071-0194. Ukr. J. Phys. 2020. Vol. 65, No. 10 
where $\eta^{2}=L^{2} / \alpha$. Equation (18) is a single equation for $b_{\mathrm{P}}(y)$ at different $\eta$. This dependence has the form of a curve with minimum. The real transition to the domain state in DMS occurs, when $T_{k}$ reaches its maximal value as a function of $y$. This, similarly to [10], can be demonstrated by the substitution of a solution of the nonlinear equation (14) in the form of an infinite series in a small parameter proportional to $\left|T-T_{k}\right|$ in the free energy (1) with its subsequent minimization over $y$. Since, for both models, $b_{\mathrm{P}}$ is a decreasing function of the temperature (e.g., for both models in the mean field approximation, $b=1-1 / \tau$, $\tau=T / T_{\mathrm{cMF}}$, see Refs. $\left.[15,16]\right)$, the coordinates of the minima $b_{\mathrm{P}}^{\min }$ and $y^{\mathrm{min}}$ of the curve $b(y)$ determine the equilibrium temperature of a phase transition to the domain state $T_{k}$ and the equilibrium period for an emerging domain structure $\lambda=2 \pi / y^{\text {min }}$ as functions of the dimensionless sample thickness $\eta$.

We calculated the minimum of the function $b(y)$ (18) numerically to get the dependences $\lambda(\eta)$ and $b_{\mathrm{P}}^{\min }(\eta)$. They are reported on the right panel of Fig. 2. It is seen that the dependence $b_{\mathrm{P}}^{\min }(\eta)$ decays rapidly as $\eta \rightarrow \rightarrow \infty$, at $\eta \rightarrow 0 b_{\mathrm{P}}^{\mathrm{min}} \rightarrow \infty$. It can be shown that, at large $\eta$,

$\lambda \propto \sqrt{\eta}=\sqrt{L / \sqrt{\alpha}}$.

This behavior is typical of a stripe domain structure in ordered magnets (see Refs. $[10,13])$ and is seen in Fig. 2.

\section{Discussion and Experimental Implications}

Now, we consider the explicit dependences $b_{\mathrm{P}}(\nu, \tau)$, $\nu=n_{c} / n_{i}=x_{c} / x_{i}, x_{c, i}=n_{c, i} \Omega, \Omega$ is the DMS unit cell volume, $n_{c}$ and $n_{i}$ are the concentrations of charge carriers (electrons or holes) and magnetic ions, respectively. Using dimensionless variables, we have

$$
\begin{aligned}
& b_{\mathrm{P}}=1-\frac{\tau}{36 \pi^{2} \nu^{2}} \int_{0}^{\infty} \mathcal{B}_{1 / 2}^{\mathrm{P}}\left(\frac{\xi \tau}{12 \nu}\right) \Phi(\xi) d \xi, \\
& \Phi(\xi)=\exp \left[\frac{\varphi_{0}(\xi)}{6 \pi \nu}\right] \varphi_{1}(\xi), \quad \mathrm{P}=\mathrm{H}, \mathrm{I},
\end{aligned}
$$

where $\xi=J_{0} x_{c}^{4 / 3} t / 2 T$. In expressions (20), we used the RKKY potential in the simplest possible form corresponding to a one-band carrier structure:

$J(\mathbf{r})=-J_{0} x_{c}^{4 / 3} F\left(2 k_{f} r\right), F(x)=\frac{x \cos x-\sin x}{x^{4}}$,
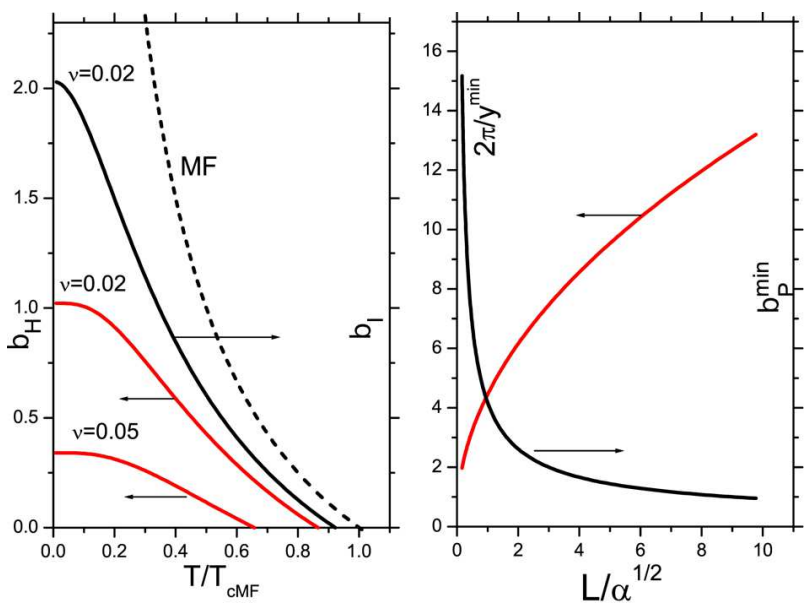

Fig. 2. Left panel: dependence of $b_{\mathrm{H}}$ and $b_{\mathrm{I}}$ on the normalized temperature at different $\nu$. Points, where $b_{\mathrm{P}}=0$, correspond to $\tau_{c}(\nu)$. Dashed line labeled MF corresponds to the case of ordered magnet $b_{\mathrm{MF}}=1-1 / \tau$. Right panel: equilibrium period of the emerging domain structure and the parameter $b$ versus the dimensionless sample thickness at $\mu_{\perp}=10$

where $J_{0}=(3 / \pi)^{1 / 3}\left(3 / 2 \hbar^{2}\right) J_{\text {ci }}^{2} \Omega^{2 / 3} m_{d}, \quad J_{\text {ci }}$ is a carrier-ion exchange constant, and $m_{d}$ is the effective mass. Functions $\varphi_{0}(\xi)$ and $\varphi_{1}(\xi)$ have the form

$\varphi_{0}(\xi)+i \varphi_{1}(\xi)=\int_{0}^{\infty}\{1-\exp [-i \xi F(y)]\} y^{2} d y$

For potential (21), the expression for $T_{\mathrm{cMF}}$ assumes the form, which will be used in subsequent numerical calculations

$T_{\mathrm{cMF}}=\frac{1}{24 \pi} J_{0} x_{i}^{4 / 3} \nu^{1 / 3}$.

The dependences $b_{\mathrm{H}}(\tau)$ and $b_{\mathrm{I}}(\tau)$ at different $\nu$ are reported in the left panel of Fig. 2. In the mean field $(\mathrm{MF})$ approximation, $b_{\mathrm{HMF}}=b_{\mathrm{IMF}}=b_{\mathrm{MF}}=1-1 / \tau$ is unbounded at $T=0$, while, beyond this approximation, these functions are finite at $T=0$

$b_{\mathrm{H} 0}=\frac{2}{3} b_{\mathrm{I} 0}=1-\frac{2}{9 \pi^{2} \nu} \int_{0}^{\infty} \Phi(\xi) \frac{d \xi}{\xi}$.

These finite values are, indeed, "responsible" for the emergence of a concentration-dependent threshold sample thickness $L_{\mathrm{tr}}$ for DMS. Having dependences (20), we can solve them numerically for $b_{\mathrm{P}}^{\min }$ 


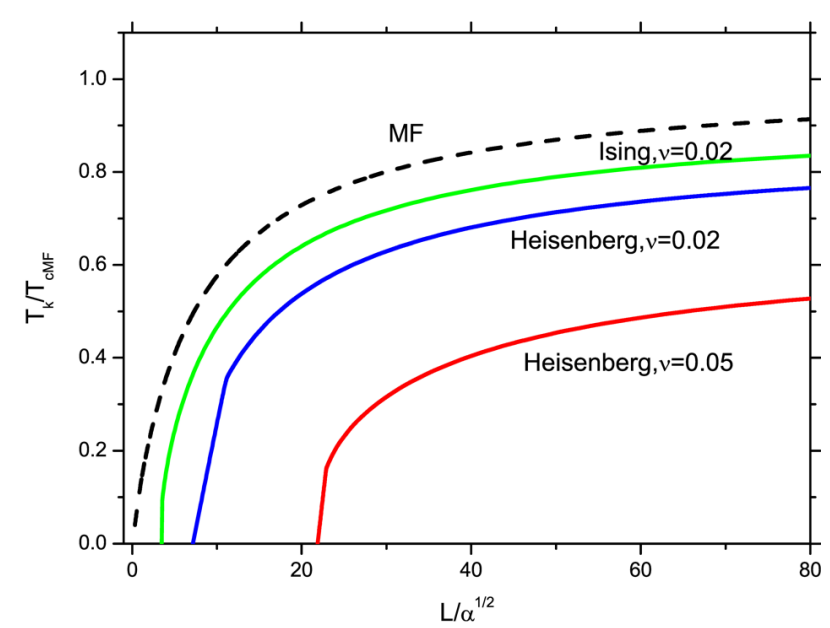

Fig. 3. Phase diagram of a DMS slab in the coordinates $T, L$. Horizontal asymptotes $-\tau_{c}(\nu)$ similar to Fig. $1, \mu_{\perp}=10$

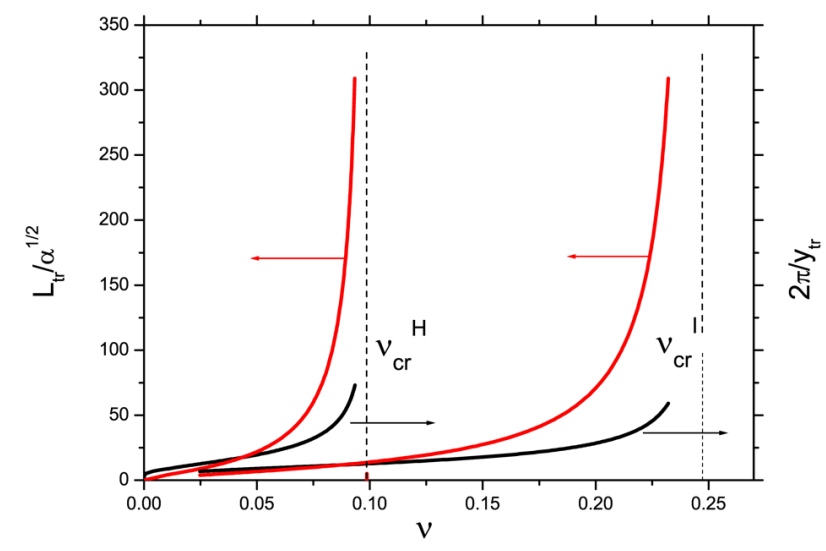

Fig. 4. Threshold sample thickness at $T=0$ and the corresponding domain structure period as functions of the concentration ratio $\nu$ at $\mu_{\perp}=10$. Critical ratios $\nu_{\mathrm{cr}}^{\mathrm{H}}$ for the Heisenberg model and $\nu_{\mathrm{cr}}^{\mathrm{I}}$ for the Ising one are shown. In the mean field approximation (ordered magnet), $\nu_{\mathrm{cr}} \rightarrow \infty$

to obtain the dimensionless phase transition temperature $\tau_{k}==T_{k} / T_{\mathrm{cMF}}$ as a function of the critical sample thickness. In other words, we have the phase diagram of a DMS slab in the coordinates $(T, L)$, which is reported in Fig. 3.

The presence of $L_{\mathrm{cr}}(\nu)$ is clearly seen. The asymptotes for large $L_{\mathrm{cr}}$ are due to the dependence of the equilibrium (corresponding to the ferromagnetic phase without domain structure) phase transition temperature $\tau_{c}=T_{c} / T_{\mathrm{cMF}}$ on the concentration ratio $\nu$. The last dependence is given by the conditions $b_{\mathrm{H}}=0$ and $b_{\mathrm{I}}=0$, Eqs. (3) and (6). It had been

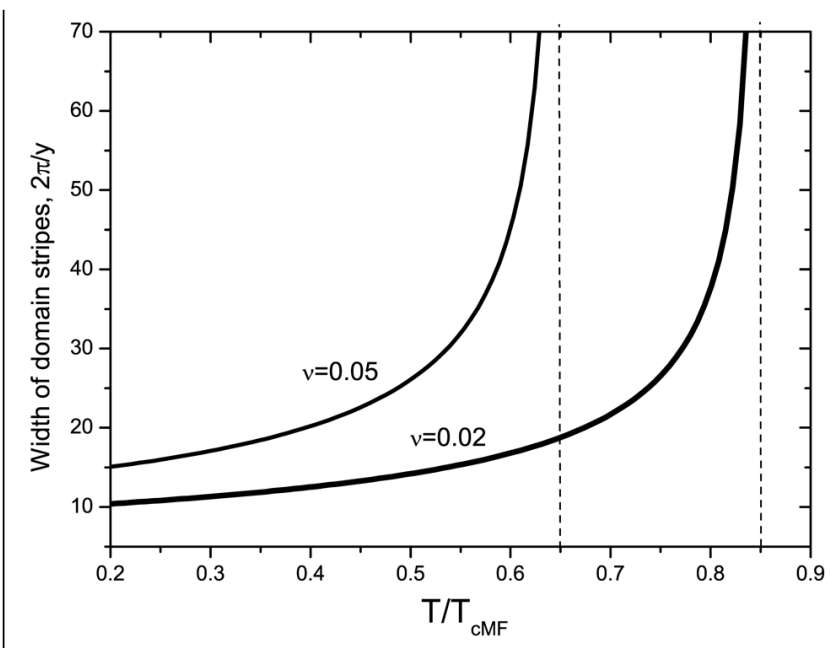

Fig. 5. Temperature dependence of the domain structure period. Critical divergence of the period at $\tau \rightarrow \tau_{c}(\nu)$ is seen. Vertical asymptotes correspond to $\tau_{c}(0.05) \approx 0.65$ and $\tau_{c}(0.02) \approx 0.85$ respectively

shown in Ref. [16] that the impurity ferromagnetism in DMS is possible for $0<\nu<\nu_{\mathrm{cr}}^{\mathrm{H}, \mathrm{I}}$,

$\nu_{\mathrm{cr}}^{\mathrm{H}}=0.0989, \quad \nu_{\mathrm{cr}}^{\mathrm{I}}=0.2473$,

so that $\tau_{c}\left(\nu_{\mathrm{cr}}\right)=0$. This means that $\tau_{c}$ decays as $\nu$ grows and the region $(T, L)$, where a ferromagnetic domain state exists in DMS, diminishes substantially (compared to the case of ordered ferromagnets) and vanishes as $\nu \rightarrow \nu_{\mathrm{cr}}$.

Note that, in the MF approximation, it is very easy to solve (20) analytically to get $\tau_{\mathrm{cMF}}=1 /\left(1-b_{\mathrm{P}}\right)$. Resolving Eqs. (23) for $b_{\mathrm{P}}^{\mathrm{min}}$, we obtain the dependence of the threshold thickness on the concentration ratio $\nu$. This dependence along with the corresponding period of the domain structure is portrayed in Fig. 4 for the Heisenberg and Ising models. Our analysis shows that the dependence of the sample critical thickness on the parameter $\nu$ (Fig. 4) has critical character and can be well approximated by the function

$$
\frac{L_{\mathrm{tr}}}{\sqrt{\alpha}}=\frac{a}{\left|\nu-\nu_{\mathrm{cr}}^{\mathrm{P}}\right|^{b_{\mathrm{P}}}},
$$

where $\nu_{\mathrm{cr}}^{\mathrm{P}}$ is defined by Eq. (24) for the Heisenberg and Ising models, respectively; $b_{\mathrm{I}} \approx 1.4, b_{\mathrm{H}} \approx 1.15$. At the same time, $a \approx 1.2$ is the same for both models. It is seen from Fig. 4 and from Eq. (25) that, as $\nu$ approaches $\nu_{\mathrm{cr}}, L_{\mathrm{tr}} \rightarrow \infty$ so that the DMS sample loses 
its ferromagnetism (both homogeneous and inhomogeneous). The domain structure period also diverges as $\nu \rightarrow \nu_{\text {cr }}$.

The temperature dependence of the equilibrium domain structure period (width of domain stripes) is shown in Fig. 5. The abrupt increase of the width of domain stripes as $T \rightarrow T_{c}$ is seen. This fact coincides with experimental results of Ref. [8]. Near $\tau_{c}$, we can get the following analytic result:

$\lambda=\frac{2 \pi}{y}=\frac{a(\nu)}{\tau-\tau_{c}(\nu)}$,

where, for the Heisenberg model and $\nu=0.05, a(\nu) \approx$ 10.1 ; for $\nu=0.02, a(\nu) \approx 8.6$.

The above divergences make it possible to control the critical thickness of a DMS sample by changing $\nu$. This, in turn, might give a possibility to design the domain structure in nanocrystals of DMS, which is useful for many technical applications, see Refs. [3, 5, $7,14]$ and references therein. Note that our formalism permits one to calculate $\nu_{\text {cr }}$ and other characteristics of a domain structure for a wide temperature range (away from $T_{c}$ ) and any sample geometry.

We are now in a position to make some numerical estimations. The major problem here is the uncertain value of the inhomogeneous exchange constant $\alpha$. It can be estimated by the expression (see Ref. [9]) $\alpha \approx k_{\mathrm{B}} T_{c} a^{2} /\left(M_{s} \mu_{0}\right)$, where $a=0.4 \mathrm{~nm}$ is a typical value of the lattice constant for DMS, $M_{s} \approx 50 \mathrm{mT}$ (Ref. [14]) is a saturation magnetization (of localized spin moments) of DMS, $T_{c}=T_{\mathrm{cMF}} \approx 100 \mathrm{~K}$ (Ref. [14]) is the temperature of a transition to the homogeneous ferromagnetic state in the mean field approximation, $k_{\mathrm{B}}$ and $\mu_{0}$ are the Boltzmann constant and the Bohr magneton, respectively. The evaluation gives $\alpha^{1 / 2} \sim 20 \mathrm{~nm}$. From Fig. 4 for $\nu=0.075$, we have the threshold sample thickness $L_{\mathrm{tr}} \sim 50 \alpha^{1 / 2}=$ $=1000 \mathrm{~nm}=1 \mu \mathrm{m}$ and the corresponding domain structure period is $\sim 25 \alpha^{1 / 2}=500 \mathrm{~nm}=0.5 \mu \mathrm{m}$ for the Heisenberg model. The same values for the Ising model occur at $\nu \sim 0.2$. These values are in fair agreement with results of Ref. [7]. Moreover, for different $\nu$, we have quite different values of $L_{\mathrm{tr}}$ and $y_{\mathrm{tr}}$. This is the base for the above-discussed domain structure engineering. For our picture to give the quantitative description of experiments with real DMS, the precise experimental determination of the inhomogeneous exchange constant $\alpha$ and the anisotropy constant $\beta$ is highly desirable.

\section{Conclusions}

The main physical message of the present paper is that the diluted magnetic semiconductors possess a huge corps of physical properties, which are absent both in "ordinary" (i.e. without magnetic impurities) semiconductors and in disordered paramagnets. One of the main points here is that the disorder in the subsystem of localized magnetic moments of DMS yields a phase transition to the ferromagnetic phase $[2,3,5,7,8]$, which can be used in many device applications. Such domain structure engineering is facilitated in DMS, since, varying the type and concentration of localized spins (as well as other defects and impurities), we can manipulate the characteristics of a domain structure to tailor them to specific needs.

Here, we have presented a formalism for the calculation of properties of the domain structure in DMS. The above results on the DMS phase diagram is the simplest application of the formalism. Generally, it permits one to calculate all desired properties of a domain structure (like the temperature and concentration dependences of the domain structure period and domain walls thickness) in the entire temperature range, as well as to account for more complex thin slab sample geometries. The latter can be accomplished by applying different boundary conditions from Eq. (13). The external magnetic field can also be easily taken into account. However, far from $T_{k}$, the solution of the resulting nonlinear differential equations would require numerical methods.

1. A.V. Komarov, S.M. Ryabchenko, O.V. Terletskii, I.I. Zheru, R.D. Ivanchuk. Magneto-optical studies and the double opticomagnetic resonance of the exciton band in $\mathrm{Mn} / 2+/-$ doped CdTe. Sov. Phys. JETP 46, 318 (1977).

2. E.A. Pashitskii, S.M. Ryabchenko. Magnetic ordering in semiconductors with magnetic impurities. Fiz. Tverd. Tela 21, (1979), 545; [Sov. Phys. Solid State 21, 322 (1979)].

3. T. Dietl, H. Ohno. Dilute ferromagnetic semiconductors: Physics and spintronic structures. Rev. Mod. Phys. 86, 187 (2014).

4. Introduction to the Physics of Diluted Magnetic Semiconductors. Edited by J. Kossut, J.A. Gaj (Springer, 2010).

5. M. Tanaka. Ferromagnet (MnAs)/III-V semiconductor hybrid structures. Semicond. Sci. Tech. 17, 327 (2002).

6. T. Dietl. Ferromagnetic semiconductors. Semicond. Sci. Tech. 17, 377 (2002).

7. T. Dietl, J. König, A.H. MacDonald. Magnetic domains in III-V magnetic semiconductors. Phys. Rev. B 64, 241201 (2001). 
8. T. Shono, T. Hasegawa, T. Fukumura, F. Matsukura, H. Ohno. Observation of magnetic domain structure in a ferromagnetic semiconductor ( $\mathrm{Ga}, \mathrm{Mn})$ As with a scanning Hall probe microscope. Appl. Phys. Lett. 77, 1363 (2000).

9. L.D. Landau, E.M. Lifshitz. Electrodynamics of Continuous Media (Wiley, 1984).

10. V.V. Tarasenko, E.V. Chenskii, I.E. Dikshtein. Theory of inhomogeneous magnetic states in ferromagnetic substances in vicinity of phase-transitions of 2nd kind. $Z h$. Eksp. Teor. Fiz. 70, 2178 (1976) [Sov. Phys. JETP 43, 1136 (1976)].

11. L.D. Landau, E.M. Lifshitz. On the theory of the dispersion of magnetic permeability in ferromagnetic bodies. Phys. Zs. UdSSR, 8, 153 (1935).

12. M.Ya. Shirobokov. On the mechanism of magnetization of ferromagnetics. Zh. Eksp. Teor. Fiz. 15, 57 (1945).

13. V.G. Bar'yakhtar, B.A. Ivanov. Phase diagram of a ferromagnetic plate in an external magnetic field. Zh. Eksp. Teor. Fiz. 72, 1504 (1977) [Sov. Phys. JETP 45, 789 (1977)].

14. T. Dietl, H. Ohno, F. Matsukura. Hole-mediated ferromagnetism in tetrahedrally coordinated semiconductors. Phys. Rev. B 63, 195205 (2001).

15. Yu. Semenov, V. Stephanovich. Enhancement of ferromagnetism in uniaxially stressed dilute magnetic semiconductors. Phys. Rev. B 67195203 (2003)

16. Yu.G. Semenov, V.A. Stephanovich. Suppression of carrierinduced ferromagnetism by composition and spin fluctuations in diluted magnetic semiconductors. Phys. Rev. B 66 075202 (2002).
17. L.N. Bulaevskii, V.L. Ginzburg. Temperature dependence of the shape of the domain wall in ferromagnetics and ferroelectrics. Zh. Eksp. Teor. Fiz. 45, 772 (1963).

Received 09.07.20

В.А. Стефанович, Ю.Г. Семенов

ВЛАСТИВОСТІ МАГНІТНОї

ДОМЕННОЇ СТРУКТУРИ В РОЗРІДЖЕНИХ МАГНІТНИХ НАПІВПРОВІДНИКАХ

$\mathrm{P}$ е $з$ ю м е

Представлено аналіз утворення доменної структури у феромагнітній фазі розріджених магнітних напівпровідників (РМН) p-типу. Аналіз виконується на підставі функціонала магнітної вільної енергії РМН, обчисленої нами раніше. Ця вільна енергія, якщо замістити РМН (невпорядкований магнетик) ефективною впорядкованою речовиною, дозволяє застосувати стандартний феноменологічний підхід до обчислення доменної структури. Використовуючи систему рівнянь Максвелла та рівняння мінімуму функціонала вільної енергії, ми показуємо наявність критичного відношення $\nu_{\text {cr }}$ концентрації носіїв заряду та магнітних іонів, при якому критична товщина $L_{\mathrm{cr}}$ розходиться при $\nu \rightarrow \nu_{\mathrm{cr}}$. При $\nu>\nu_{\text {cr }}$ кристал $є$ монодоменним. Ця особливість відрізняє РМН від звичайних упорядкованих магнетиків, оскільки дає можливість змінювати критичну товщину шляхом зміни $\nu$. Представлену теорію можна легко узагальнити для довільного невпорядкованого магнетика. 